\title{
EDITORIAL
}

Special Focus Issue: Pain Management in Fibromyalgia

\section{The role of diet in the treatment of fibromyalgia}

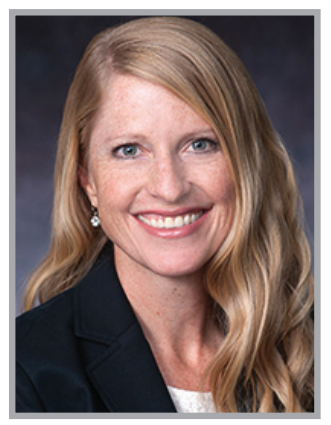

Kathleen Holton*

\author{
“...good biological plausibility exists for how \\ dietary excitotoxins may be leading to increased \\ symptom occurrence in fibromyalgia.”
}

First draft submitted: 7 April 2016; Accepted for publication: 13 May 2016; Published online: 14 June 2016

Fibromyalgia (FM) patients suffer from chronic overlapping symptoms including widespread pain, fatigue, cognitive dysfunction and sleep problems, which are thought to be related to central sensitization [1]. To date, no treatment alone provides extensive symptom relief, which leads patients to wonder whether or not diet, as something directly in their control, could be modulated to lead to improvement. Currently, there is accumulating evidence that diet may be contributing to symptoms in FM, with strong biological plausibility for how specific dietary components may be leading to abnormal glutamatergic neurotransmission, perpetuating the process of central sensitization.

Chronic pain is thought to be mediated by an initial noxious stimulus which causes release of the excitatory amino acid glutamate, which then acts on AMPA receptors on second order neurons. Prolonged activation of nociceptors leads to continuous release of glutamate, which releases the magnesium block in NMDA receptors, and combined with the release of substance $P$, can cause long-lasting membrane depolarization leading to chronic pain [2]. In addition to glutamate's role in central sensitization, abnormal glutamatergic neurotransmission has also been implicated in other common overlapping pain conditions like migraine [3], temporomandibular joint disorder [4] and irritable bowel syndrome [5], in addition to common comorbid mood disorders like depression [6]. Glutamate is an excitatory neurotransmitter, with the job of perpetuating action potentials (electrical signals) in both the brain and the periphery. However, if glutamate is released in high enough amounts, it also has the ability to overexcite postsynaptic neurons to the point that they die. This process is called excitotoxicity $[7]$.

Substance $\mathrm{P}$, which is co-released with glutamate during nociception, has been shown to increase the permeability of the blood-brain barrier (BBB) [8,9]. The BBB normally protects the brain from high plasma concentrations of dietary glutamate [10]. Thus, increased concentrations of substance P observed during central sensitization, may be leading to increased $\mathrm{BBB}$ permeability, which could in turn

*Department of Health Studies, Center for Behavioral Neuroscience, American University, 4400 Massachusetts Avenue NW, Gray Hall 119, Washington, DC 20016, USA; Tel.: +1 202885 3797; holton@american.edu

\section{KEYWORDS}

- antioxidants - chronic pain

- excitotoxicity - fibromyalgia

- glutamate $\bullet$ magnesium $\bullet$ omega-3 fatty acids $\bullet$ vitamin $\mathrm{B} 6 \bullet$ zinc

Future
Medicine
part of 


\section{"Future research attempting to modulate glutamatergic neurotransmission should also include measurement of the dietary intake and plasma levels of these micronutrients, since there \\ is great potential for interaction between these nutrients and excitotoxicity."}

allow dietary glutamate to enter the brain more readily, influencing symptom occurrence in FM.

Multiple studies have demonstrated a link between glutamate and pain occurrence. Injection of glutamate into the masseter muscle in the jaw has been shown to induce pain in healthy subjects [11]. Higher cerebrospinal fluid glutamate concentrations have been demonstrated in FM and migraine patients as compared with healthy controls [12]. Furthermore, higher brain glutamate levels have been reported in multiple studies utilizing magnetic resonance spectroscopy in FM patients as compared with healthy controls [13].

From a dietary perspective, glutamate is one of two negatively charged, nonessential, amino acids (glutamate and aspartate) in the diet which also function as excitatory neurotransmitters. Aspartate acts on the NMDA receptor, which is a key glutamate receptor implicated in pain and depression [14]. Glutamate and aspartate are found in bound and free forms in the diet. Bound forms include full protein sources like meat, where amino acids are released slowly into circulation during the digestive process. In contrast, free forms of these amino acids are found in the diet as food additives (such as monosodium glutamate [MSG], hydrolyzed protein, protein isolates/concentrates, yeast extract, aspartame [a dipeptide of phenylalanine and aspartate] and others) [15], as well as in specific food items such as soy sauce, fish sauces and aged cheeses like sharp cheddar and parmesan cheese [16,17]. Theoretically, higher plasma concentrations could lead to abnormal excitation in the PNS, and in people with an impaired BBB, this could also theoretically lead to abnormal excitation in the central nervous system.

In 2001, Smith et al. reported on a case series of four subjects whose FM symptoms improved after removing MSG and aspartame from their diets [18]. Another case series in 2010 reported remission of FM symptoms and other widespread pain in two patients who stopped using aspartame, with return of symptoms after initiating use of aspartame again [19]. A clinical trial later demonstrated significant symptom improvement in FM patients who were put on an excitotoxin elimination diet for 1 month (removing all sources of the free forms of these amino acids), with eight subjects experiencing complete remission of all symptoms [20]. Subjects who reported $>30 \%$ of their symptoms remitting after 1 month on the diet, were eligible to go onto a randomized, double-blind, placebo-controlled crossover challenge with MSG and placebo, to test whether symptom recurrence could be induced. Results demonstrated a significant return of symptoms upon challenge with MSG as compared with placebo, suggesting that the elimination of excitotoxins was the reason for symptom relief [20]. In contrast, another study by Vellisca and Latorre reported no overall improvement after a 3-month exclusion of MSG and aspartame in FM patients [21]. A significant reduction in pain was noted after 1 month, but no difference between groups was evident in the second and third months. Little details were given about training and dietary adherence of study participants, nor were details provided on whether or not the placebo group could have obtained information on what the intervention group was doing. Therefore, the results seen at the end of the first month could have been diminished over the next 2 months by either: decreased dietary adherence or by contamination of the placebo group. Furthermore, subjects were not instructed to avoid all dietary excitotoxins, but rather only MSG and aspartame; thus, potential benefit would depend on whether or not the main sources of exposure were removed from the diet. Overall, there is good biological plausibility for how dietary glutamate and aspartate could potentially modulate neurotransmission in FM patients. Therefore, future research examining the potential mechanism behind the excitotoxin elimination diet is warranted.

The only way to test for sensitivity to excitotoxins is to completely remove this exposure from the diet. Generally, this consists of eating a whole food diet which limits the consumption of foods with additives. In addition to avoiding overt ingredients such as MSG, aspartame and altered proteins (like gelatin, hydrolyzed protein, autolyzed yeast extract, protein concentrates and protein isolates), patients should also look for ingredient lists on food labels which are short, easy to read, and which only include ingredients that a person could add to food when cooking. For example, certain terms such as spices, seasoning, flavoring, natural flavoring, among others. should also be avoided since these terms are not specific as to what is included in the food and could hide these additives. Mixed seasoning packets commonly contain flavor enhancers like MSG and thus, should be replaced with whole herbs and spices when cooking. Additionally, foods naturally high in free glutamate including soy sauce, fish sauces, Worcestershire sauce, Bragg's amino acids and cheeses like parmesan should 
also be avoided. To avoid aspartame, it is important to avoid diet soda, gum and breath mints, and special care must be taken in selecting items like yogurt, cereal and bread, as artificial sweeteners are currently being added to many foods since they are hundreds of times sweeter than sugar. During the testing period, foods should only be sweetened naturally with table sugar or honey. A 1-month testing period (without eating out) is ideal, though in prior research most subjects started to feel an improvement by the end of the first week.

Micronutrients also play an important role in supporting optimal neuronal functioning, and a few nutrients in particular have the ability to potentially modulate glutamatergic neurotransmission. Two minerals in the diet play a key role in regulating the NMDA receptor, the main glutamate receptor implicated in excitotoxicity. Magnesium blocks the NMDA receptor and must be removed in order for excitation to occur [22]. Additionally, zinc is co-released with glutamate into the synaptic cleft, and is thought to negatively modulate the excitatory response [23]. Thus, low magnesium and zinc levels could support excitotoxicity.

Another micronutrient of importance to glutamatergic neurotransmission is vitamin B6. This vitamin serves as an important cofactor for the enzyme glutamate decarboxylase, which converts glutamate (an excitatory neurotransmitter) into GABA (an inhibitory neurotransmitter) [24]. Deficiency in B6 can lead to higher levels of glutamate and reduced levels of GABA inhibition, thereby facilitating excitotoxicity in the central nervous system.

Deficiency in omega-3 fatty acids has been shown to increase excitotoxicity [25]. This effect is thought to be mediated by the ability of omega-3 fatty acids to increase fluidity of the cell membrane, which could potentially modulate the expression of key glutamate transporters, which are responsible for clearing excess glutamate from the synaptic cleft, thereby preventing excitotoxicity.

\section{References}

Papers of special note have been highlighted as:

- of interest; $\bullet$ of considerable interest

1 Clauw DJ. Fibromyalgia and related conditions. Mayo Clin. Proc. 90(5), 680-692 (2015).

2 Chizh BA. Novel approaches to targeting glutamate receptors for the treatment of
In addition to the above direct nutrient effects, there may also be an important downstream role for micronutrients. Excitotoxicity can cause oxidative stress through increased production of reactive oxygen species in the nervous system [26]. Oxidative stress can be countered through the dietary intake of antioxidants [27]. Thus, excessive glutamatergic neurotransmission leads to increased need for antioxidants in the diet. The two main vitamin antioxidants in the diet are vitamin C (a water soluble antioxidant) and vitamin $\mathrm{E}$ (a fat soluble antioxidant), though other chemicals found in fruits and vegetables have also been shown to have important antioxidant capabilities, such as resveratrol in grapes [28] and polyphenols in green tea [29].

In conclusion, good biological plausibility exists for how dietary excitotoxins may be leading to increased symptom occurrence in FM. Low intake of omega-3 fatty acids, vitamin B6, magnesium, zinc and antioxidants may also play a supporting role in symptom occurrence. Future research attempting to modulate glutamatergic neurotransmission should also include measurement of the dietary intake and plasma levels of these micronutrients, since there is great potential for interaction between these nutrients and excitotoxicity. Research is also needed using a combined intervention approach which modulates intake of excitotoxins while concurrently increasing the consumption of these key nutrients, to examine the potential for synergistic effects.

\section{Financial \& competing interests disclosure}

The author has no relevant affliations or financial involvement with any organization or entity with a financial interest in or financial conflict with the subject matter or materials discussed in the manuscript. This includes employment, consultancies, honoraria, stock ownership or options, expert testimony, grants or patents received or pending, or royalties.

No writing assistance was utilized in the production of this manuscript. chronic pain: review article. Amino Acids 23(1-3), 169-176 (2002).

3 Fang J, An X, Chen S, Yu Z, Ma Q, Qu H. Case-control study of GRIA1 and GRIA3 gene variants in migraine. J. Headache Pain 17(1), 2 (2015).

4 Castrillon EE, Ernberg M, Cairns BE et al. Interstitial glutamate concentration is elevated in the masseter muscle of myofascial temporomandibular disorder patients. J. Orofac. Pain. 24(4), 350-360 (2010).

5 Niddam DM, Tsai SY, Lu CL, Ko CW, Hsieh JC. Reduced hippocampal glutamateglutamine levels in irritable bowel syndrome: preliminary findings using magnetic 
resonance spectroscopy. Am. J. Gastroenterol. 106(8), 1503-1511 (2011).

6 Kim YK, Na KS. Role of glutamate receptors and glial cells in the pathophysiology of treatment-resistant depression. Prog. Neuropsychopharmacol. Biol. Psychiatry doi:10.1016/j.pnpbp.2016.03.009 (2016) (Epub ahead of print).

7 Mehta A, Prabhakar M, Kumar P, Deshmukh R, Sharma PL. Excitotoxicity: bridge to various triggers in neurodegenerative disorders. Eur. J. Pharmacol. 698(1-3), 6-18 (2013).

-• Great review on excitotoxicity.

8 Rodriguez PL, Jiang S, Fu Y, Avraham S, Avraham HK. The proinflammatory peptide substance P promotes blood-brain barrier breaching by breast cancer cells through changes in microvascular endothelial cell tight junctions. Int. J. Cancer 134(5), 1034-1044 (2014).

9 Dossantos MF, Holanda-Afonso RC, Lima RL, Dasilva AF, Moura-Neto V. The role of the blood-brain barrier in the development and treatment of migraine and other pain disorders. Front. Cell. Neurosci. 8, 302 (2014).

- Good review of potential role of blood-brain barrier in pain disorders.

10 Hawkins RA. The blood-brain barrier and glutamate. Am. J. Clin. Nutr. 90(3), S867-S874 (2009).

11 Shimada A, Cairns BE, Vad N et al. Headache and mechanical sensitization of human pericranial muscles after repeated intake of monosodium glutamate (MSG). J. Headache Pain 14, 2 (2013).

12 Peres MF, Zukerman E, Senne Soares CA, Alonso EO, Santos BF, Faulhaber MH. Cerebrospinal fluid glutamate levels in chronic migraine. Cephalalgia 24(9), 735-739 (2004).
13 Harris RE. Elevated excitatory neurotransmitter levels in the fibromyalgia brain. Arthritis Res. Ther. 12(5), 141 (2010).

14 Potter WZ, Brady LS. Current drug development for antidepressants and ideas addressing downstream glutamate: the ketamine example. CNS Spectr. 19(2), 112-114 (2014).

15 Blaylock RL. Excitotoxins: the Taste that Kills. Health Press, Santa Fe, NM, USA (1997).

16 Hajeb P, Jinap S. Umami taste components and their sources in Asian foods. Crit. Rev. Food Sci. Nutr. 55(6), 778-791 (2015).

17 Ochi H, Sakai Y, Koishihara H, Abe F, Bamba T, Fukusaki E. Monitoring the ripening process of Cheddar cheese based on hydrophilic component profiling using gas chromatography-mass spectrometry. J. Dairy Sci. 96(12), 7427-7441 (2013).

18 Smith JD, Terpening CM, Schmidt SO, Gums JG. Relief of fibromyalgia symptoms following discontinuation of dietary excitotoxins. Ann. Pharmacother. 35(6), 702-706 (2001).

19 Ciappuccini R, Ansemant T, Maillefert JF, Tavernier C, Ornetti P. Aspartame-induced fibromyalgia, an unusual but curable cause of chronic pain. Clin. Exp. Rheumatol. 28(6 Suppl. 63), S131-S133 (2010).

20 Holton KF, Taren DL, Thomson CA, Bennett RM, Jones KD. The effect of dietary glutamate on fibromyalgia and irritable bowel symptoms. Clin. Exp. Rheumatol. 30(6 Suppl. 74), 10-17 (2012).

- Relevant example of dietary intervention for testing for sensitivity to excitotoxins.

21 Vellisca MY, Latorre JI. Monosodium glutamate and aspartame in perceived pain in fibromyalgia. Rheumatol. Int. 34(7), 1011-1013 (2014).

22 Savic Vujovic KR, Vuckovic S, Srebro D et al. A synergistic interaction between magnesium sulphate and ketamine on the inhibition of acute nociception in rats. Eur. Rev. Med. Pharmacol. Sci. 19(13), 2503-2509 (2015).

23 Anderson CT, Radford RJ, Zastrow ML et al. Modulation of extrasynaptic NMDA receptors by synaptic and tonic zinc. Proc. Natl Acad. Sci. USA 112(20), E2705-E2714 (2015).

24 Amadasi A, Bertoldi M, Contestabile R et al. Pyridoxal 5'-phosphate enzymes as targets for therapeutic agents. Curr. Med. Chem. 14(12), 1291-1324 (2007).

25 Keleshian VL, Kellom M, Kim HW et al. Neuropathological responses to chronic NMDA in rats are worsened by dietary n-3 PUFA deprivation but are not ameliorated by fish oil supplementation. PLoS ONE 9(5), e95318 (2014).

26 Borkum JM. Migraine triggers and oxidative stress: a narrative review and synthesis. Headache 56(1), 12-35 (2016).

27 Wang JY, Wen LL, Huang YN, Chen YT, Ku MC. Dual effects of antioxidants in neurodegeneration: direct neuroprotection against oxidative stress and indirect protection via suppression of glia-mediated inflammation. Curr. Pharm. Des. 12(27), 3521-3533 (2006).

28 Zhang Q, Yuan L, Zhang Q et al. Resveratrol attenuates hypoxia-induced neurotoxicity through inhibiting microglial activation. Int. Immunopharmacol. 28(1), 578-587 (2015).

29 Cong L, Cao C, Cheng Y, Qin XY. Green tea polyphenols attenuated glutamate excitotoxicity via antioxidative and antiapoptotic pathway in the primary cultured cortical neurons. Oxid. Med. Cell Longev. 2016, 2050435 (2016).

- May be of interest for potentially recommending the consumption of green tea as a source of antioxidants. 